BOOK REVIEW

\section{The regulation of assisted reproductive technology}

Edited by J Gunning, H Szoke. Ashgate, 2003, pp 271. ISBN 0754622924.

This book brings together papers given at a symposium which took place in Melbourne, Australia in 2001. Like any such collection, the chapters vary in quality and in substance. Some authors have chosen to analyse one issue in considerable depth, while others attempt a broad overview of regulations in different regions of the world. Julian Savulescu, for example, confines himself to the complex question of whether gamete providers' freedom to dispose of their unwanted embryos should take priority over infertile couples' interests in using these surplus embryos in treatment. In contrast, Jothi Kumar surveys the regulation of assisted reproductive technology throughout Asia in a mere four pages of text. In general, contributions of the first type tend to be more successful; attempts to summarise something as complex as the regulation of assisted reproductive technology across a whole continent can be difficult for the reader to digest, and inevitably offer little by way of analysis

A further problem bedevils any new publication in this fast moving area of medical law and ethics. Although providing a "snapshot" of regulation in 2001 is undoubtedly an interesting and worthwhile project, a book such as this risks being out of date before it is even published. As a result, the more discursive chapters which concentrate on especially novel or controversial ethical dilemmas are likely to be of use and interest for longer than those which describe the regulatory status quo. Certainly, Savulescu's chapter on the public interest in embryos, and Giuliana Fuscaldo's contribution on the relevance and value of genetic ties will probably have a longer shelf life than others which will inevitably be overtaken by events.

Nevertheless, some of the chapters which attempt a regulatory overview do offer especially interesting insights into different countries' approaches to regulation in this area. Ruth Deech, who was Chair of the UK's Human Fertilisation and Embryology Authority (HFEA) from 1994-2002, provides an admirably clear and succinct account of the HFEA's first 10 years. Her summary of the social and legal background to the UK's much admired (and copied) regulatory framework is especially useful. Since her chapter was written there have, of course, been further developments. In particular, there have been a number of legal actions against the Authority, and several sections of the Human Fertilisation and Embryology Act have been subjected to intensive judicial scrutiny. There have also been several high profile IVF "blunders"- such as the case in which a woman's eggs were fertilised with the wrong man's sperm-which have forced the HFEA to tighten up its regulatory and licensing procedures, as well as resulting in some complicated and previously unanticipated questions about legal fatherhood.

Two well written and interesting chapters, by Guido Pennings and Kristen Walker respectively, tackle the complex question of whether it is legitimate to take the future child's welfare into account when deciding whether to offer a particular couple or individual access to assisted reproductive technology. Pennings addresses this issue in the context of would-be parents who might be at risk of passing on a genetic disease to their offspring, but his clear and thoughtful analysis could undoubtedly have a wider resonance. He suggests three different versions of a welfare based criterion. The first would require doctors to maximise children's wellbeing, meaning that fertility treatment should only be provided where the future child's life conditions would be optimal. This, he argues, is too restrictive and would in practice rule out almost all potential parents. The second is a minimum threshold standard, under which fertility treatment should be available to anyone, unless their future child would have a quality of life so low that it would be better for them not to have been born. Hardly any potential parents will fail this test, which effectively amounts to a presumption in favour of universal access. Pennings criticises this test on the grounds that it endows non-existent potential lives with interests in existence that can be harmed by denying their "parents" access to fertility treatment-as if all possible future people are waiting in an existential vestibule until the choice about whether or not they should be conceived is taken.

Pennings' preferred solution is his third version: treatment should be provided as long as the child is likely to have a reasonably fulfilling life. But, of course, the problem of deciding when a future child's quality of life is likely to be unacceptably low remains. Here Pennings points out some interesting inconsistencies in prevailing attitudes towards acceptable and unacceptable risks. The adoption of precautions such as antiretroviral drugs, caesarean delivery, and abstention from breastfeeding can reduce the risk of vertical HIV transmission to $1-2 \%$, yet HIV positive individuals are commonly refused access to fertility treatment. The increased mortality faced by twins $(4.7 \%)$ is higher than this, and yet not believed a good reason to deny patients access to treatment in which two embryos are transferred in one cycle. Nor is a factor such as a parent's reduced life expectancy applied consistently between different categories of patients. HIV positive individuals or postmenopausal women will often be denied treatment, whereas intracytoplasmic sperm injection (ICSI) is used to treat infertility in male cystic fibrosis patients despite their increased risk of premature death.

Kristen Walker's perceptive chapter contrasts two different mechanisms for invoking the future child's welfare as a criterion with which to limit access to assisted reproductive services. The first mechanism is that there could be a general direction-as in the UKto clinicians to take account of the welfare of any child who may be born before providing a woman with treatment services. Walker rejects this approach on the grounds that it is inherently vague and subjective. If a couple do not yet have any children, working out their probable parental aptitude with any degree of accuracy and fairness would be prohibitively time consuming and expensive. Nor, she argues, is it clear that doctors are particularly well equipped to make this sort of judgement. The second model she considers is a presumption of access, subject to a list of excluded categories of individualssuch as anyone with a conviction for child abuse, or with an addiction to drugs or alcohol. Although this approach has the merit of clarity and transparency, it too (as Walker points out) may be unsatisfactory. Even the most apparently straightforward exclusion of individuals with previous convictions for child abuse might lead to injustice. Would we, Walker asks, want to exclude a 40 year old man who, when aged 19, had a sexual relationship with a 15 year old girl, and who has had no other convictions?

The contributions of Fuscaldo and Savulescu offer thought provoking and critical accounts of couples' attitudes towards their embryos stored in vitro. Both authors question the importance of genetic ties, and advocate donation rather than disposal of surplus embryos. Fuscaldo looks at the reasons why couples decide to dispose of embryos in preference to donation, and argues that they are based upon a series of misunderstandings about the importance of genetic relatedness. Savulescu argues that couples whose surplus embryos are stored in vitro are under a duty of "easy rescue". Donating those embryos to infertile couples poses no risk of harm or inconvenience to their wellbeing or interests. In contrast, the benefit that could accrue to recipient couples is enormous. In practice, Savulescu proposes an opt out system, similar to that advocated in related to organ transplants, in which donation rather than disposal is the default solution.

This book offers a number of very interesting contributions to the ongoing debates over the appropriate scope of the regulation of assisted reproductive technologies. It will undoubtedly be a useful addition to any library. One minor quibble, however, is the sheer number of typographical errors, and some inelegant indecisiveness within chapters over the appropriate spelling of words such as centre/center.

E Jackson e.jackson@|se.ac.uk 